\title{
A Novel Method for Constructing Grid Multi-Wing Butterfly Chaotic Attractors via Nonlinear Coupling Control
}

\author{
Yun Huang \\ Asset Management Department, Chongqing University of Posts and Telecommunications, Chongqing 400065, China \\ Correspondence should be addressed to Yun Huang; hyztt1@sohu.com
}

Received 19 April 2016; Revised 23 August 2016; Accepted 29 August 2016

Academic Editor: George S. Tombras

Copyright (C) 2016 Yun Huang. This is an open access article distributed under the Creative Commons Attribution License, which permits unrestricted use, distribution, and reproduction in any medium, provided the original work is properly cited.

\begin{abstract}
A new method is presented to construct grid multi-wing butterfly chaotic attractors. Based on the three-dimensional Lorenz system, two first-order differential equations are added along with one linear coupling controller, respectively. And a piecewise linear function, which is taken into the linear coupling controller, is designed to form a nonlinear coupling controller; thus a fivedimensional chaotic system is produced, which is able to generate gird multi-wing butterfly chaotic attractors. Through the analysis of the equilibrium points, Lyapunov exponent spectrums, bifurcation diagrams, and Poincaré mapping in this system, the chaotic characteristic of the system is verified. Apart from the research above, an electronic circuit is designed to implement the system. The circuit experimental results are in accordance with the results of numerical simulation, which verify the availability and feasibility of this method.
\end{abstract}

\section{Introduction}

Since Lorenz found the first chaotic model in 1963 [1], people have had a great interest to construct the chaotic attractors with different shape and quantity. At present, the existing chaotic attractors could be divided into two types: the multiscroll chaotic attractors (including double-scroll [2], multiscroll [3, 4], gird multi-scroll [5], and multi-directional multiscroll chaotic attractors [6]) and the multi-wing butterfly chaotic attractors (containing two-wing [7-11], four-wing [12-16], multi-wing [17-20], and grid multi-wing [21, 22] butterfly chaotic attractors). The approach had grown pretty mature constructing multi-scroll chaotic attractors. However, it is rarely researched for the method constructing multi-wing and grid multi-wing butterfly chaotic attractors.

In recent years, [17-22] reported the latest research achievement about the multi-wing and grid multi-wing butterfly chaotic attractors. Reference [17] constructed a class of hyperchaotic systems generating $2^{n}$-wing butterfly hyperchaotic attractors by coordinate transition and absolute value transition. References $[18,21]$ proposed the grid multiwing butterfly chaotic attractors by constructing heteroclinic loops. Reference [7] presented the first and second kinds of Lorenz-type systems to simplify the algebraic form of the
Lorenz system while keeping the butterfly structure of Lorenz attractor. Reference [19] constructed a multi-wing butterfly chaotic system based on the first and second kinds of Lorenztype systems. By designing some piecewise functions to take place of the state variables of the Lorenz system directly, $[20,22]$ proposed some multi-wing and grid multi-wing butterfly chaotic systems. Though the above literatures proposed some methods constructing multi-wing and grid multi-wing butterfly chaotic attractors, it is very difficult to construct the new multi-wing and grid multi-wing butterfly chaotic attractors via these approaches. Therefore, it is necessary to design the novel methods constructing multi-wing and grid multi-wing butterfly chaotic attractors.

In this paper, through combining a linear coupling controller with a piecewise linear function skillful, a new method is presented to construct grid multi-wing butterfly chaotic attractors. Based on the three-dimensional Lorenz system, two first-order differential equations are added along with one linear coupling controller, respectively. And a piecewise linear function, which is taken into the linear coupling controller, is designed to form a nonlinear coupling controller; thus a five-dimensional chaotic system is produced, which is able to generate gird multi-wing butterfly chaotic attractors. 
The system is easy to be realized by analog circuits and its algebraic form is also simple.

\section{Constructing the Grid Multi-Wing Butterfly Chaotic Attractors via the Nonlinear Coupling Control}

The mathematic model of Lorenz chaotic system is shown as follows:

$$
\begin{aligned}
& \dot{x}_{1}=a\left(x_{2}-x_{1}\right), \\
& \dot{x}_{2}=c x_{1}-x_{2}-x_{1} x_{3}, \\
& \dot{x}_{3}=x_{1} x_{2}-b x_{3},
\end{aligned}
$$

where $a=10, b=8 / 3$, and $c=28$.

Constructing grid multi-wing butterfly chaotic attractors via the nonlinear coupling control, the method is shown as follows.

Step 1. In order to construct gird multi-wing butterfly chaotic attractors easier, the state variables need normalization processing in system (1). The method of the normalization processing is to make the peak value of the all state variables less than one via scale transformation. Let the scaling factors of the state variables $x_{1}, x_{2}$ and $x_{3}$ be $\gamma_{1}, \gamma_{2}$, and $\gamma_{3}$, respectively. According to the numerical simulation results of the Lorenz system [1], we can choose $\gamma_{1}=16, \gamma_{2}=23$, and $\gamma_{3}=40$. Therefore, system (1) is changed as follows:

$$
\begin{aligned}
& \dot{X}_{1}=\frac{a \gamma_{2}}{\gamma_{1}} X_{2}-a X_{1}, \\
& \dot{X}_{2}=\frac{c \gamma_{1}}{\gamma_{2}} X_{1}-X_{2}-\frac{\gamma_{1} \gamma_{3}}{\gamma_{2}} X_{1} X_{3}, \\
& \dot{X}_{3}=\frac{\gamma_{1} \gamma_{2}}{\gamma_{3}} X_{1} X_{2}-b X_{3} .
\end{aligned}
$$

Step 2. Two first-order differential equations about state variables $X_{4}$ and $X_{5}$ are added based on system (2). The exact approach is as follows: First, the first and second equations are copied and taken as the fourth and fifth equations in system (2), and then the state variable $X_{1}$ is replaced with $X_{4}$ in the fourth equation of system (2), and the state variable $X_{5}$ is used to take place of the state variable $X_{2}$ in the fifth equation of system (2). Therefore, a five-dimensional autonomous system is obtained:

$$
\begin{aligned}
& \dot{X}_{1}=\frac{a \gamma_{2}}{\gamma_{1}} X_{2}-a X_{1}, \\
& \dot{X}_{2}=\frac{c \gamma_{1}}{\gamma_{2}} X_{1}-X_{2}-\frac{\gamma_{1} \gamma_{3}}{\gamma_{2}} X_{1} X_{3}, \\
& \dot{X}_{3}=\frac{\gamma_{1} \gamma_{2}}{\gamma_{3}} X_{1} X_{2}-b X_{3}, \\
& \dot{X}_{4}=\frac{a \gamma_{2}}{\gamma_{1}} X_{2}-a X_{4}, \\
& \dot{X}_{5}=\frac{c \gamma_{1}}{\gamma_{2}} X_{1}-X_{5}-\frac{\gamma_{1} \gamma_{3}}{\gamma_{2}} X_{1} X_{3} .
\end{aligned}
$$

Step 3. Two linear coupling controllers $u_{1}$ and $u_{2}$ are added in the fourth and fifth equations of system (3), respectively. Then system (3) is changed as follows:

$$
\begin{aligned}
& \dot{X}_{1}=\frac{a \gamma_{2}}{\gamma_{1}} X_{2}-a X_{1} \\
& \dot{X}_{2}=\frac{c \gamma_{1}}{\gamma_{2}} X_{1}-X_{2}-\frac{\gamma_{1} \gamma_{3}}{\gamma_{2}} X_{1} X_{3}, \\
& \dot{X}_{3}=\frac{\gamma_{1} \gamma_{2}}{\gamma_{3}} X_{1} X_{2}-b X_{3}, \\
& \dot{X}_{4}=\frac{a \gamma_{2}}{\gamma_{1}} X_{2}-a X_{4}+u_{1} \\
& \dot{X}_{5}=\frac{c \gamma_{1}}{\gamma_{2}} X_{1}-X_{5}-\frac{\gamma_{1} \gamma_{3}}{\gamma_{2}} X_{1} X_{3}+u_{2},
\end{aligned}
$$

where the coupling controllers $u_{1}=h\left(X_{1}-X_{4}\right)$ and $u_{2}=$ $h\left(X_{2}-X_{5}\right) . h$ is gain parameter.

Step 4. A new piecewise linear function is designed:

$$
f(x)=x-\sum_{n=-N}^{M} \operatorname{sgn}(x+2 n+1)+(M-N+1),
$$

where $N, M \in\{0,1,2, \ldots\}$. Through replacing the state variables $X_{4}$ and $X_{5}$ with the piecewise linear function $f(x)$ in system (4), a novel system is obtained as follows:

$$
\begin{aligned}
& \dot{X}_{1}=a_{1} X_{2}-a_{2} X_{1}, \\
& \dot{X}_{2}=a_{3} X_{1}-X_{2}-a_{4} X_{1} X_{3}, \\
& \dot{X}_{3}=a_{5} X_{1} X_{2}-a_{6} X_{3}, \\
& \dot{X}_{4}=a_{1} X_{2}-a_{2} f\left(X_{4}\right)+u_{1}^{\prime}, \\
& \dot{X}_{5}=a_{3} X_{1}-f\left(X_{5}\right)-a_{4} X_{1} X_{3}+u_{2}^{\prime},
\end{aligned}
$$

where the system parameter $a_{1}=a \gamma_{2} / \gamma_{1}, a_{2}=a, a_{3}=c \gamma_{1} / \gamma_{2}$, $a_{4}=\gamma_{1} \gamma_{3} / \gamma_{2}, a_{5}=\gamma_{1} \gamma_{2} / \gamma_{3}, a_{6}=b$, and the nonlinear coupling controllers $u_{1}^{\prime}=h\left[X_{1}-f\left(X_{4}\right)\right]$ and $u_{2}^{\prime}=h\left[X_{2}-f\left(X_{5}\right)\right]$

$$
\begin{aligned}
f\left(X_{4}\right)= & X_{4}-\sum_{n=-N_{1}}^{M_{1}} \operatorname{sgn}\left(X_{4}+2 n+1\right) \\
& +\left(M_{1}-N_{1}+1\right), \\
f\left(X_{5}\right)= & X_{5}-\sum_{n=-N_{2}}^{M_{2}} \operatorname{sgn}\left(X_{5}+2 n+1\right) \\
& +\left(M_{2}-N_{2}+1\right),
\end{aligned}
$$

where $N_{1}, M_{1}, N_{2}, M_{2} \in\{0,1,2, \ldots\}$.

Let the system parameters $a=10, b=8 / 3, c=28$, $\gamma_{1}=16, \gamma_{2}=23, \gamma_{3}=40, h=5$; that is, $a_{1}=14.38$, 
$a_{2}=10, a_{3}=19.48, a_{4}=27.83, a_{5}=9.20, a_{6}=2.67$. Under the action of the nonlinear coupling controllers $u_{1}^{\prime}$ and $u_{2}^{\prime}$, system (6) is able to generate $2\left(N_{1}+M_{1}+2\right) \times\left(N_{2}+M_{2}+2\right)$ wing butterfly chaotic attractors, as shown in Figure 1 . When $N_{1}=M_{1}=N_{2}=M_{2}=0$, system (6) creates $4 \times 2$-wing butterfly chaotic attractors as shown in Figure 1(a). When $N_{1}=M_{1}=M_{2}=0$ and $N_{2}=1$, system (6) generates $4 \times 3$-wing butterfly chaotic attractors as shown in Figure 1(b). When $N_{1}=N_{2}=1$ and $M_{1}=M_{2}=0$, system (6) creates $6 \times 3$-wing butterfly chaotic attractors as shown in Figure 1(c), and the time series about the state variable $X_{4}$ is shown in Figure 1(e). When $N_{1}=N_{2}=M_{2}=1$ and $M_{1}=0$, system (6) generates $6 \times 4$-wing butterfly chaotic attractors as shown in Figure 1(d).

\section{Basic Dynamic Characteristic}

3.1. Equilibrium Point. Let $\dot{X}_{1}=\dot{X}_{2}=\dot{X}_{3}=\dot{X}_{4}=\dot{X}_{5}=0$; that is,

$$
\begin{array}{r}
a_{1} X_{2}-a_{2} X_{1}=0, \\
a_{3} X_{1}-X_{2}-a_{4} X_{1} X_{3}=0, \\
a_{5} X_{1} X_{2}-a_{6} X_{3}=0, \\
a_{1} X_{2}-a_{2} f\left(X_{4}\right)+u_{1}^{\prime}=0, \\
a_{3} x_{1}-f\left(x_{5}\right)-a_{4} x_{1} x_{3}+u_{2}^{\prime}=0 .
\end{array}
$$

According to solution for the equation set (8), the equilibrium points of system (6) are obtained:

$$
\begin{aligned}
& E_{n_{1} n_{2}}^{1}=\left(0,0,0,2 n_{1}, 2 n_{2}\right), \\
& E_{n_{1} n_{2}}^{2}=\left(A, \frac{A a_{2}}{a_{1}}, \frac{A^{2} a_{2} a_{5}}{a_{1} a_{6}}, A+2 n_{1}, \frac{A a_{2}}{a_{1}}+2 n_{2}\right), \\
& E_{n_{1} n_{2}}^{3} \\
& \quad=\left(-A,-\frac{A a_{2}}{a_{1}}, \frac{A^{2} a_{2} a_{5}}{a_{1} a_{6}},-A+2 n_{1},-\frac{A a_{2}}{a_{1}}+2 n_{2}\right),
\end{aligned}
$$

where $A=\sqrt{\left(a_{1} a_{3} a_{6}-a_{2} a_{6}\right) / a_{2} a_{4} a_{5}}, n_{1}=-\left(N_{1}+1\right),-N_{1}$, $\ldots, 0, \ldots, M_{1}$ and $n_{2}=-\left(N_{2}+1\right),-N_{2}, \ldots, 0, \ldots, M_{2}$.

For the equilibrium points $E^{*}=\left(X_{1}^{*}, X_{2}^{*}, X_{3}^{*}, X_{4}^{*}, X_{5}^{*}\right)$, system (6) is linearized and the Jacobian matrix is defined as

$$
=\left(\begin{array}{ccccc}
-a_{2} & a_{1} & 0 & 0 & 0 \\
a_{3}-a_{4} X_{3}^{*} & -1 & -a_{4} X_{1}^{*} & 0 & 0 \\
a_{5} X_{2}^{*} & a_{5} X_{1}^{*} & -a_{6} & 0 & 0 \\
h & a_{1} & 0 & -a_{2}-h & 0 \\
a_{3}-a_{4} X_{3}^{*} & h & -a_{4} X_{1}^{*} & 0 & -1-h
\end{array}\right),
$$

where $X_{1}^{*}, X_{2}^{*}, X_{3}^{*}, X_{4}^{*}$, and $X_{5}^{*}$ are the coordinates of the equilibrium points $E_{n_{1} n_{2}}^{1}, E_{n_{1} n_{2}}^{2}$, and $E_{n_{1} n_{2}}^{3}$.

Substituting the equilibrium points $E_{n_{1} n_{2}}^{1}=\left(0,0,0,2 n_{1}\right.$, $\left.2 n_{2}\right)$ into the characteristic equation $\operatorname{det}(\mathbf{J}-\lambda \mathbf{I})=0$, we get the following eigenvalues: $\lambda_{1}=-6.00, \lambda_{2}=-15.00, \lambda_{3}=$ -22.83, $\lambda_{4}=11.83$, and $\lambda_{5}=-2.67 . \lambda_{1}, \lambda_{2}, \lambda_{3}$, and $\lambda_{5}$ are negative real numbers, and $\lambda_{4}$ is a positive real number. So the equilibrium points $E_{n_{1} n_{2}}^{1}$ are unstable saddle.

Substituting the equilibrium points $E_{n_{1} n_{2}}^{2}=\left(A, A a_{2} / a_{1}\right.$, $\left.A^{2} a_{2} a_{5} / a_{1} a_{6}, A+2 n_{1}, A a_{2} / a_{1}+2 n_{2}\right)$ into the characteristic equation $\operatorname{det}(\mathbf{J}-\lambda \mathbf{I})=0$, we get the following eigenvalues: $\lambda_{1}=-15.00, \lambda_{2}=-6.00, \lambda_{3,4}=0.09 \pm 10.20 i$, and $\lambda_{5}=$ -13.86. $\lambda_{1}, \lambda_{2}$, and $\lambda_{5}$ are negative real numbers, and $\lambda_{3,4}$ are a pair of conjugate complex eigenvalues with positive real parts. Through computation, we know that the equilibrium points $E_{n_{1} n_{2}}^{3}$ have the same eigenvalues as the equilibrium points $E_{n_{1} n_{2}}^{2}$. Therefore, the equilibrium points $E_{n_{1} n_{2}}^{2}$ and $E_{n_{1} n_{2}}^{3}$ are unstable saddle-foci of index 2.

\subsection{Lyapunov Exponent Spectrum, Bifurcation Diagram, and} Poincaré Mapping. Let $N_{1}=N_{2}=1$ and $M_{1}=M_{2}=0$; the Lyapunov exponent of system (6) and its bifurcation diagram which varies with the coefficient $a_{6}$ and its Poincaré map are shown in Figure 2. The range of values for the coefficient $a_{6}$ is 0 to 3. Figures 2 (a) and 2(b) show that when $a_{6} \in[0.75,3]$, system (6) has one positive Lyapunov exponent, so it is in the chaotic state. From Figure 2(c), we can see that the Poincaré mapping spread out from multiple directions. This shows the complicated dynamic behavior of system (6).

\section{Circuit Design of the Grid Multi-Wing Butterfly Chaotic Attractors}

According to system (6), the circuit of grid multi-wing butterfly chaotic attractors is designed as shown in Figure 3.

Let $N_{1}=N_{2}=M_{2}=1$ and $M_{1}=0$; the piecewise linear function (7) is changed as follows

$$
\begin{aligned}
& f\left(X_{4}\right)=X_{4}-\sum_{n=-1}^{0} \operatorname{sgn}\left(X_{4}+2 n+1\right), \\
& f\left(X_{5}\right)=X_{5}-\sum_{n=-1}^{1} \operatorname{sgn}\left(X_{5}+2 n+1\right)+1 .
\end{aligned}
$$

The circuit diagram of the piecewise linear function (11) is shown in Figure 4.

In Figures 3 and 4, all the operational amplifiers are selected as UA741CN. Their supply voltage $E= \pm 15 \mathrm{~V}$ and saturated voltage $V_{\text {sat }} \approx \pm 13.5 \mathrm{~V}$. All the multipliers are of type AD633JN and their gain is $0.1 . E^{+}$is the positive pole of the supply voltage $E$; that is, $E^{+}=15 \mathrm{~V}$. $E^{-}$is the negative pole of the supply voltage $E$; that is, $E^{-}=-15 \mathrm{~V}$. 


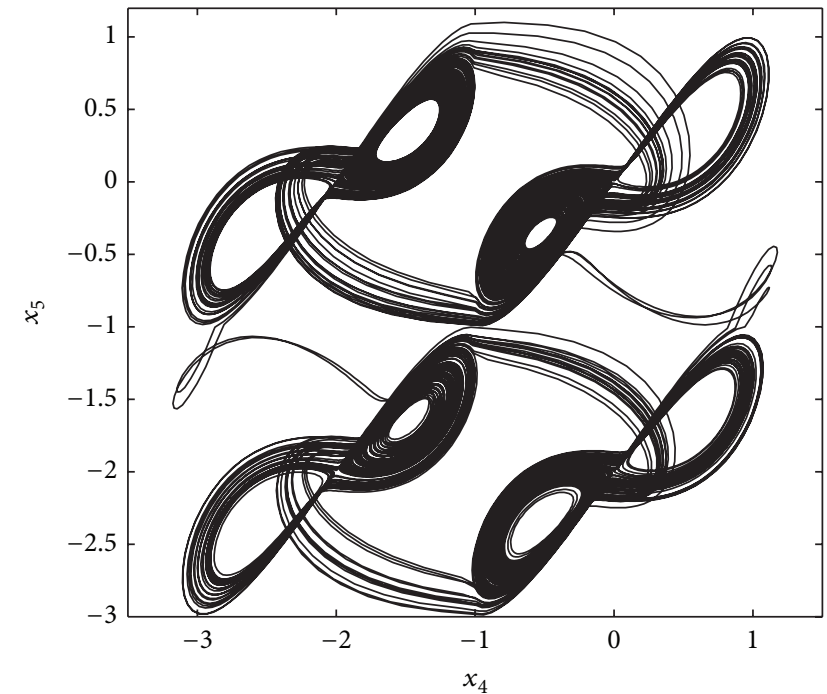

(a)

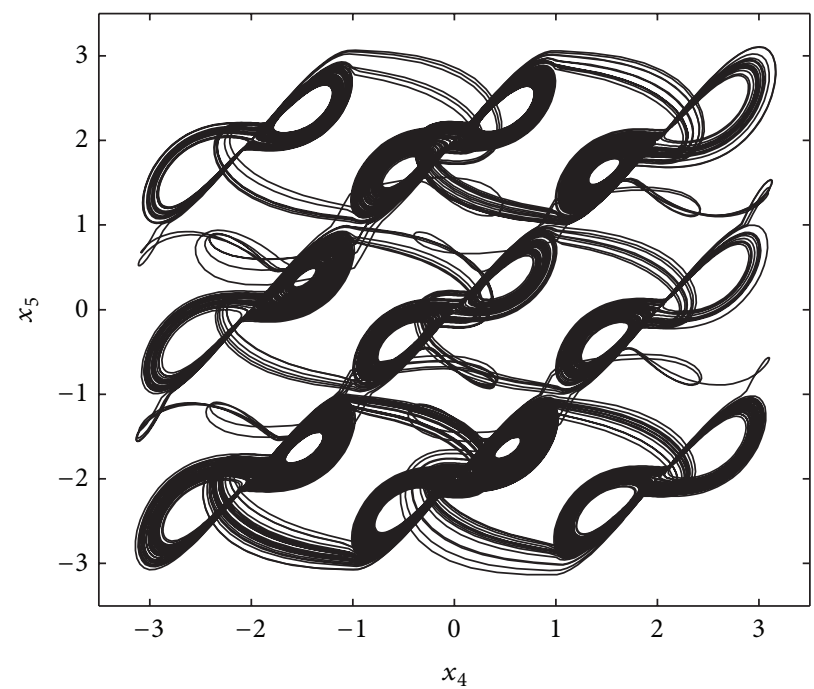

(c)

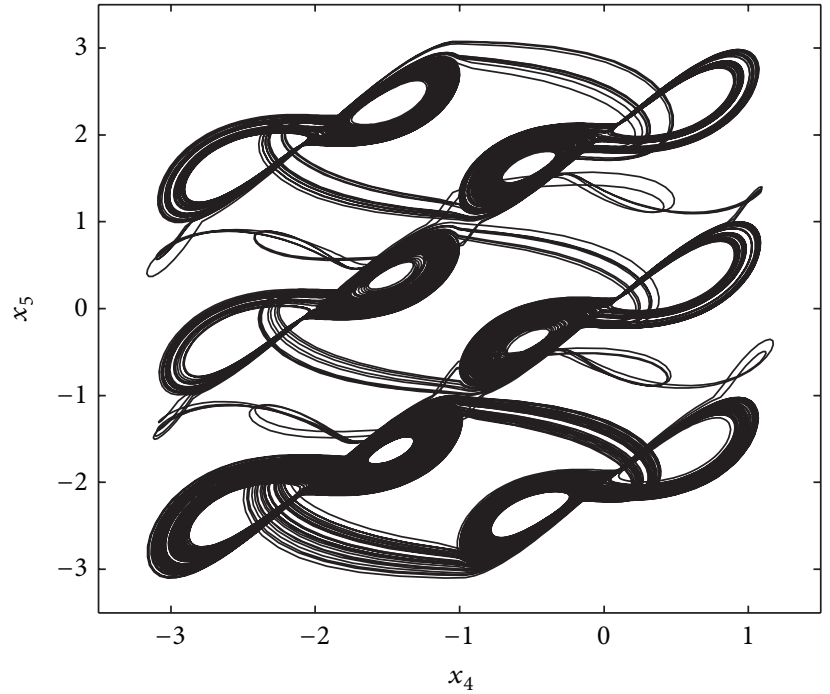

(b)

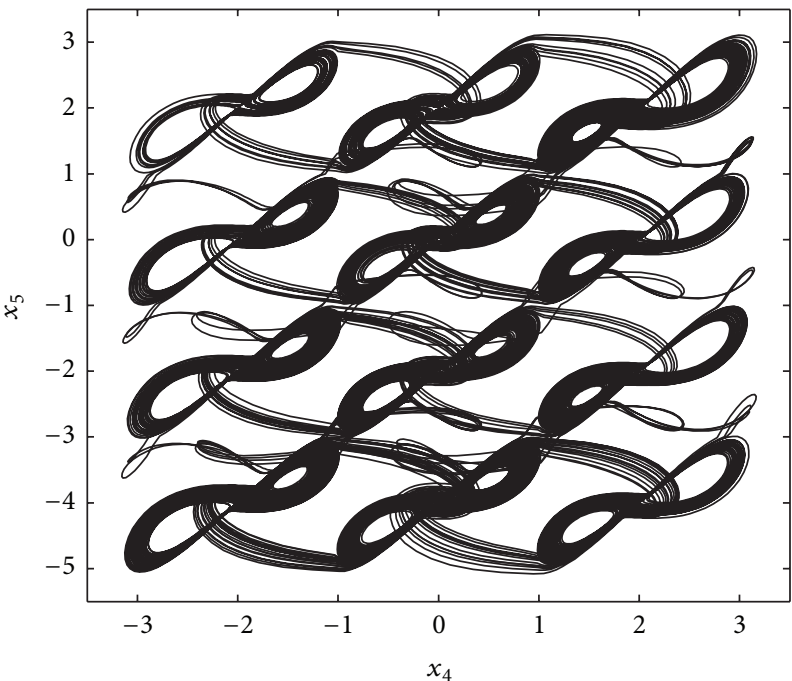

(d)

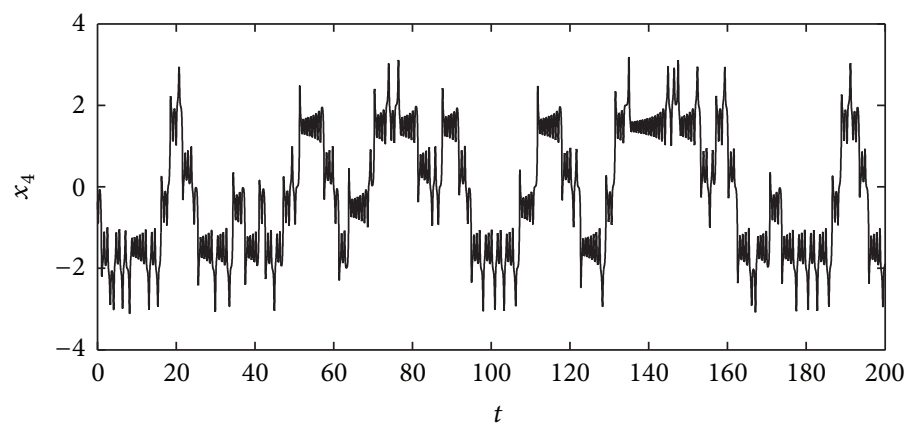

(e)

FIGURE 1: Grid multi-wing butterfly chaotic attractors and time series of system (6): (a) $4 \times 2$; (b) $4 \times 3$; (c) $6 \times 3$; (d) $6 \times 4$; (e) time series about the state variable $X_{4}$. 


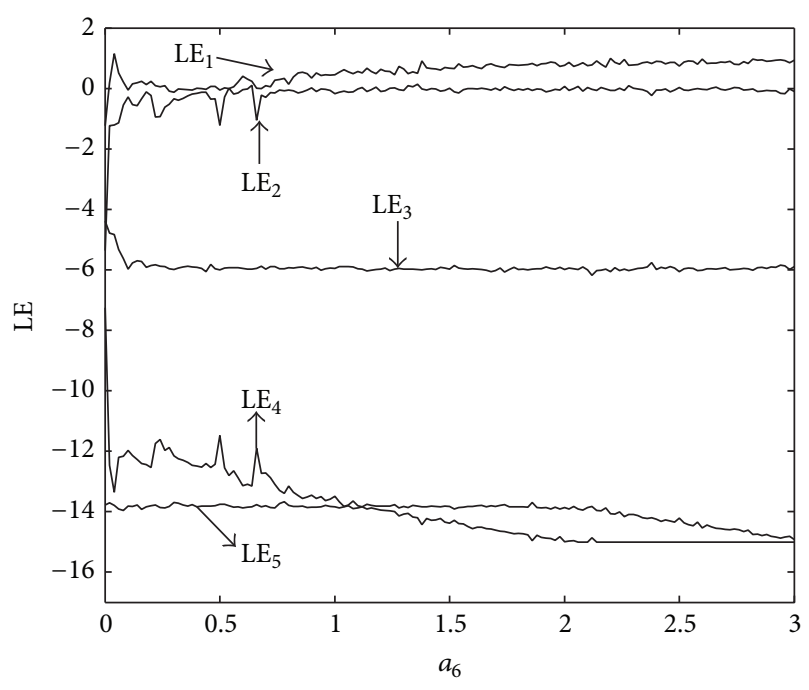

(a)

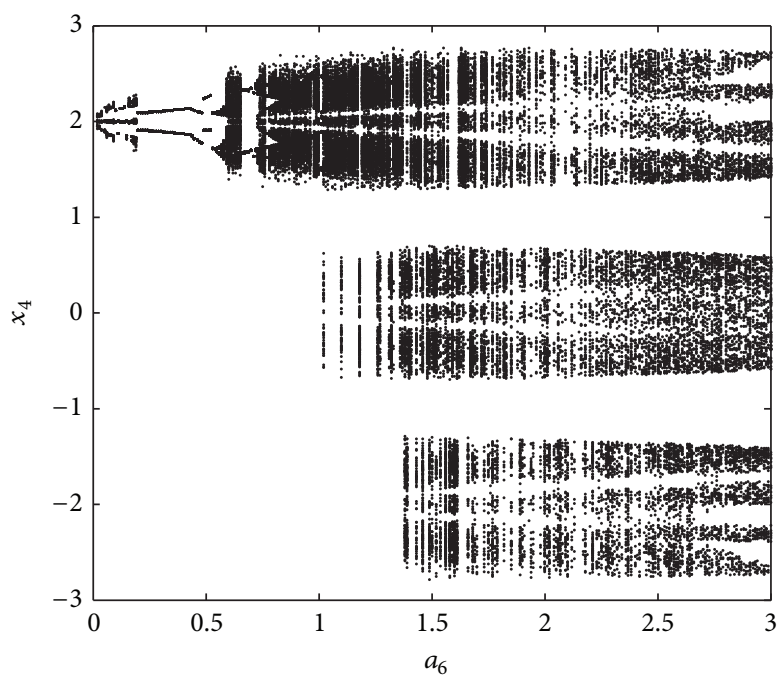

(b)

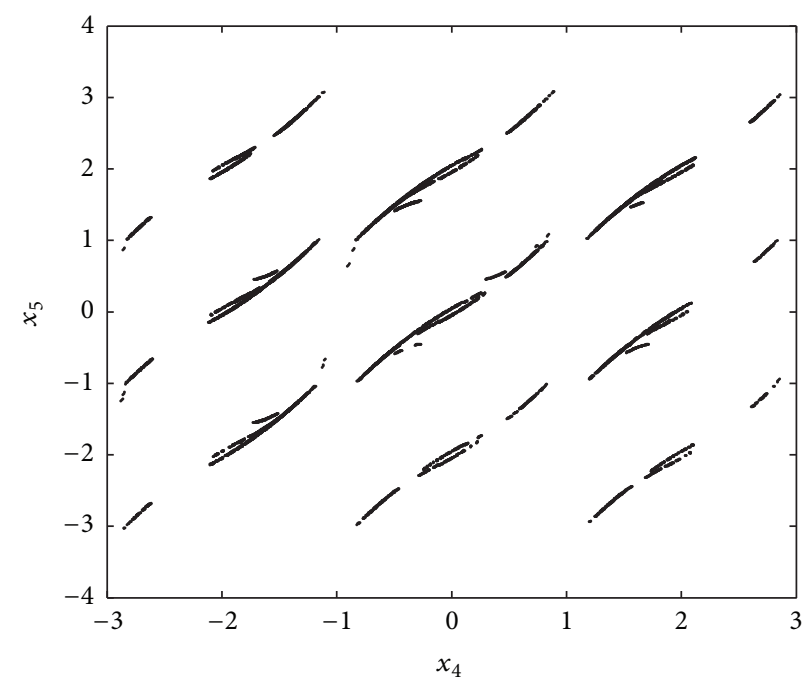

(c)

Figure 2: Lyapunov exponent spectrum, bifurcation diagram, and Poincaré mapping of system (6): (a) Lyapunov exponent spectrum; (b) bifurcation diagram; (c) Poincaré mapping.

According to Figure 3, the circuit equation can be obtained as follows:

$$
\frac{d X_{1}}{d \tau}=\frac{1}{R_{0} C_{0}}\left(\frac{R_{15}}{R_{1}} X_{2}-\frac{R_{15}}{R_{2}} X_{1}\right)
$$$$
\frac{d X_{2}}{d \tau}=\frac{1}{R_{0} C_{0}}\left(-\frac{R_{15}}{10 R_{3}} X_{1} X_{3}+\frac{R_{15}}{R_{4}} X_{1}-\frac{R_{15}}{R_{5}} X_{2}\right)
$$$$
\frac{d X_{3}}{d \tau}=\frac{1}{R_{0} C_{0}}\left(\frac{R_{15}}{10 R_{6}} X_{1} X_{2}-\frac{R_{15}}{R_{7}} X_{3}\right)
$$$$
\frac{d X_{4}}{d \tau}=\frac{1}{R_{0} C_{0}}\left(\frac{R_{15}}{R_{8}} X_{2}+\frac{R_{15}}{R_{9}} X_{1}-\frac{R_{15}}{R_{10}} f\left(X_{4}\right)\right),
$$

$$
\begin{aligned}
& \frac{d X_{5}}{d \tau}=\frac{1}{R_{0} C_{0}}\left(-\frac{R_{15}}{10 R_{11}} X_{1} X_{3}+\frac{R_{15}}{R_{12}} X_{1}+\frac{R_{15}}{R_{13}} X_{2}\right. \\
& \left.\quad-\frac{R_{15}}{R_{14}} f\left(X_{5}\right)\right) .
\end{aligned}
$$

To observe the output wave experimentally, the time scale transformation must be executed for $\tau$, that is, let $\tau=\tau_{0} t$ and $\tau_{0}=10^{-3}$, and (12) can be changed as follows:

$$
\begin{aligned}
& \frac{d X_{1}}{d \tau}=\frac{10^{-3}}{R_{0} C_{0}}\left(\frac{R_{15}}{R_{1}} X_{2}-\frac{R_{15}}{R_{2}} X_{1}\right), \\
& \frac{d X_{2}}{d \tau}=\frac{10^{-3}}{R_{0} C_{0}}\left(-\frac{R_{15}}{10 R_{3}} X_{1} X_{3}+\frac{R_{15}}{R_{4}} X_{1}-\frac{R_{15}}{R_{5}} X_{2}\right),
\end{aligned}
$$




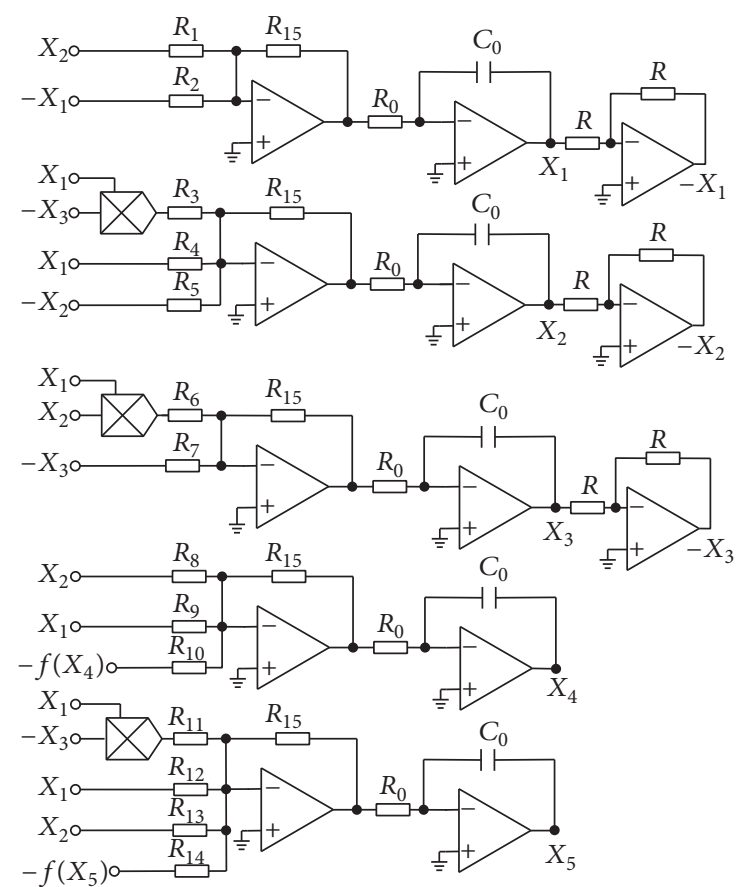

FIgURE 3: Circuit diagram of grid multi-wing butterfly chaotic attractors.

$$
\begin{aligned}
& \frac{d X_{3}}{d \tau}=\frac{10^{-3}}{R_{0} C_{0}}\left(\frac{R_{15}}{10 R_{6}} X_{1} X_{2}-\frac{R_{15}}{R_{7}} X_{3}\right), \\
& \frac{d X_{4}}{d \tau}=\frac{10^{-3}}{R_{0} C_{0}}\left(\frac{R_{15}}{R_{8}} X_{2}+\frac{R_{15}}{R_{9}} X_{1}-\frac{R_{15}}{R_{10}} f\left(X_{4}\right)\right), \\
& \frac{d X_{5}}{d \tau}=\frac{10^{-3}}{R_{0} C_{0}}\left(-\frac{R_{15}}{10 R_{11}} X_{1} X_{3}+\frac{R_{15}}{R_{12}} X_{1}+\frac{R_{15}}{R_{13}} X_{2}\right. \\
& \left.\quad-\frac{R_{15}}{R_{14}} f\left(X_{5}\right)\right) .
\end{aligned}
$$

Let $R_{0}=10 \mathrm{k} \Omega, C_{0}=10 \mathrm{nF}, R=10 \mathrm{k} \Omega$, and $R_{15}=$ $100 \mathrm{k} \Omega$; according to system (6) and equation (13), we can obtain $R_{1}=R_{8}=69.54 \mathrm{k} \Omega, R_{2}=100 \mathrm{k} \Omega, R_{3}=R_{11}=$ $3.59 \mathrm{k} \Omega, R_{4}=R_{12}=51.33 \mathrm{k} \Omega, R_{5}=1 \mathrm{M} \Omega, R_{6}=10.87 \mathrm{k} \Omega$, $R_{7}=362.32 \mathrm{k} \Omega, R_{9}=R_{13}=200 \mathrm{k} \Omega, R_{10}=66.67 \mathrm{k} \Omega$, and $R_{14}=166.67 \mathrm{k} \Omega$.

In Figure 4 , let $R_{V I}=135 \mathrm{k} \Omega$. When $k_{1}, k_{2}, k_{3}, k_{4}$, and $k_{5}$ are switched on, the circuit equation can be obtained as follows:

$$
\begin{aligned}
-f( & \left.X_{4}\right) \\
= & -\frac{R}{R} X_{4} \\
& +\frac{R}{R_{V I}}\left|V_{\text {sat }}\right| \operatorname{sgn}\left(X_{4}-\frac{R_{18}}{R_{16}+R_{17}+R_{18}} E^{-}\right) \\
& -\frac{R}{R} \cdot \frac{R_{18}}{R_{18}+R_{19}} E^{+}
\end{aligned}
$$

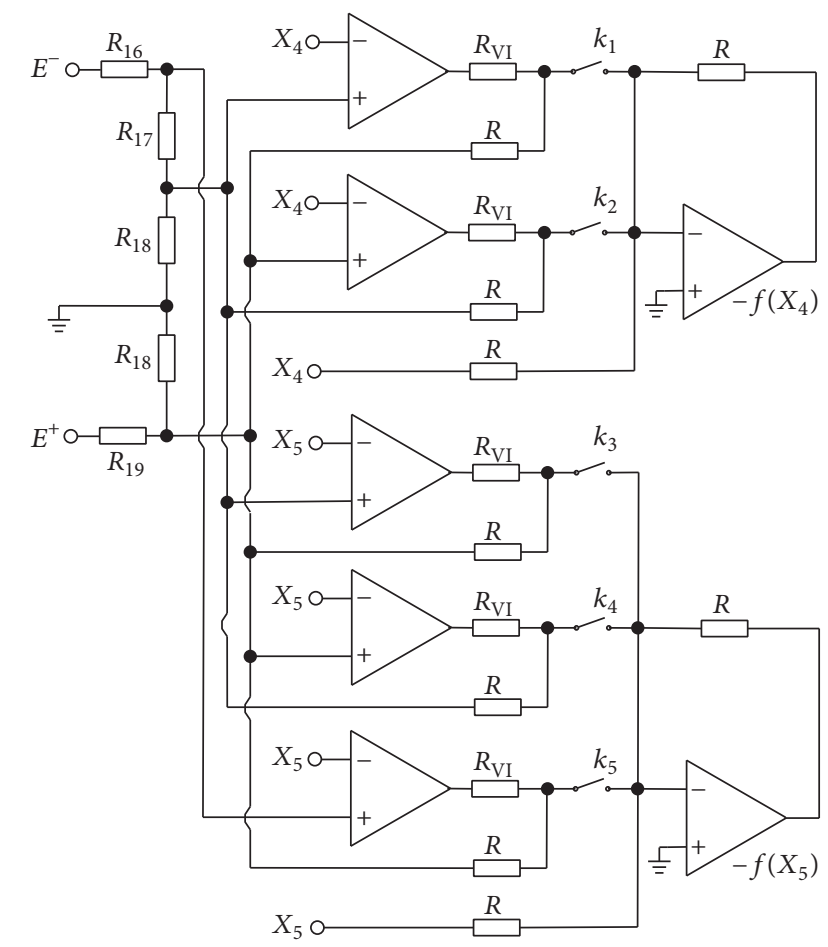

FIgURE 4: Circuit diagram of the piecewise linear function (11).

$$
\begin{aligned}
& +\frac{R}{R_{V I}}\left|V_{\text {sat }}\right| \operatorname{sgn}\left(X_{4}-\frac{R_{18}}{R_{18}+R_{19}} E^{+}\right)-\frac{R}{R} \\
& \cdot \frac{R_{18}}{R_{16}+R_{17}+R_{18}} E^{-} \\
= & -X_{4}+\operatorname{sgn}\left(X_{4}+\frac{15 R_{18}}{R_{16}+R_{17}+R_{18}}\right) \\
& -\frac{15 R_{18}}{R_{18}+R_{19}}+\operatorname{sgn}\left(X_{4}-\frac{15 R_{18}}{R_{18}+R_{19}}\right) \\
& +\frac{15 R_{18}}{R_{16}+R_{17}+R_{18}}, \\
-f & \left(X_{5}\right) \\
= & -\frac{R}{R} X_{5} \\
& +\frac{R}{R_{V I}}\left|V_{\text {sat }}\right| \operatorname{sgn}\left(X_{5}-\frac{R_{18}}{R_{16}+R_{17}+R_{18}} E^{-}\right) \\
& -\frac{R}{R} \cdot \frac{R_{18}}{R_{18}+R_{19}} E^{+} \\
& +\frac{R}{R_{V I}}\left|V_{\text {sat }}\right| \operatorname{sgn}\left(X_{5}-\frac{R_{18}}{R_{18}+R_{19}} E^{+}\right)-\frac{R}{R} \\
& \cdot \frac{R_{18}}{R_{16}+R_{17}+R_{18}} E^{-} \\
& +\frac{R}{R_{V I}}\left|V_{\text {sat }}\right| \operatorname{sgn}\left(X_{5}-\frac{R_{17}+R_{18}}{R_{16}+R_{17}+R_{18}} E^{-}\right)
\end{aligned}
$$




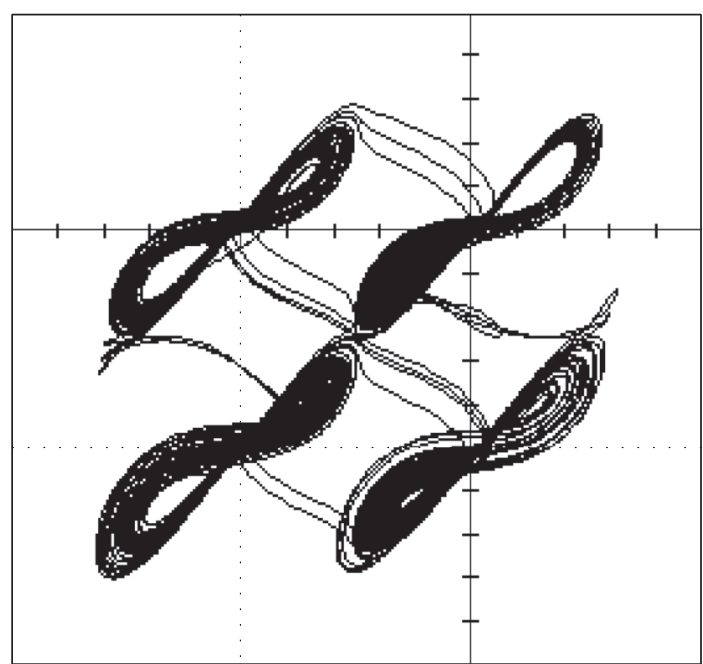

(a)

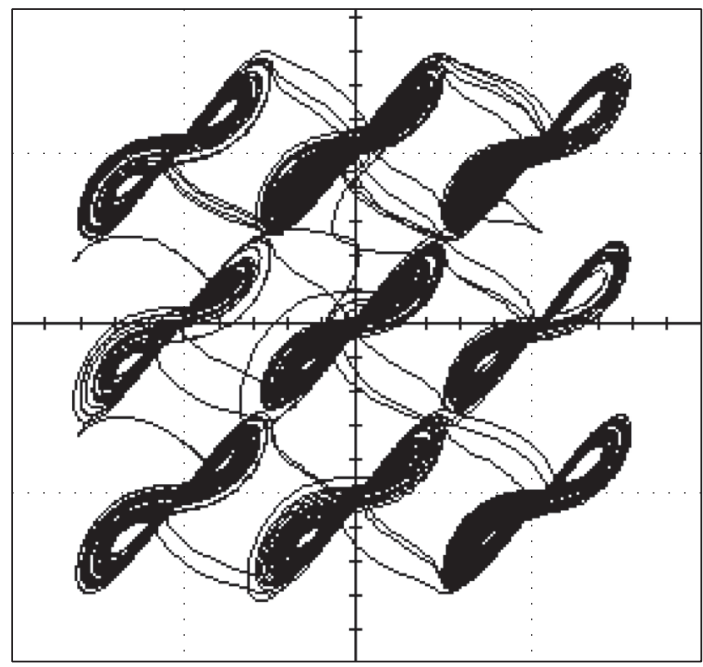

(c)

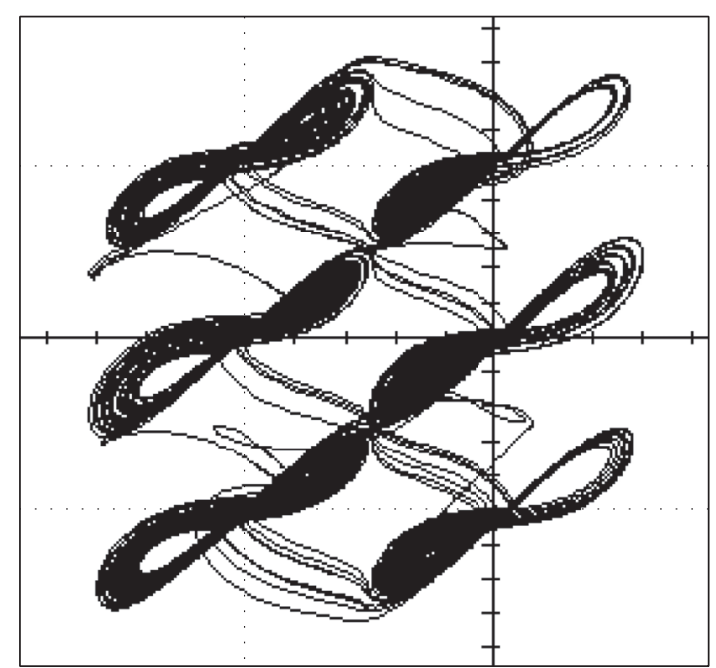

(b)

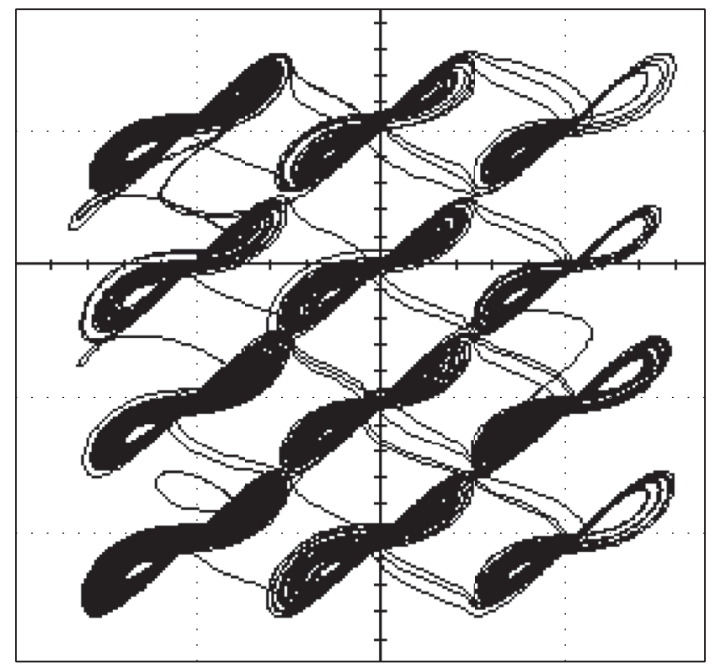

(d)

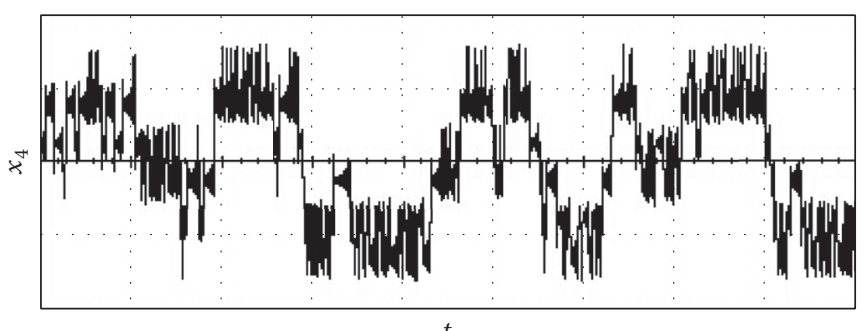

(e)

Figure 5: The results of circuit experiment: (a) $4 \times 2$; (b) $4 \times 3$; (c) $6 \times 3$; (d) $6 \times 4$; (e) time series about the state variable $X_{4}$.

$$
\begin{aligned}
& -\frac{R}{R} \cdot \frac{R_{18}}{R_{18}+R_{19}} E^{+} \\
= & -X_{5}+\operatorname{sgn}\left(X_{5}+\frac{15 R_{18}}{R_{16}+R_{17}+R_{18}}\right) \\
& -\frac{15 R_{18}}{R_{18}+R_{19}}+\operatorname{sgn}\left(X_{5}-\frac{15 R_{18}}{R_{18}+R_{19}}\right)
\end{aligned}
$$

$$
\begin{aligned}
& +\frac{15 R_{18}}{R_{16}+R_{17}+R_{18}} \\
& +\operatorname{sgn}\left(X_{5}+\frac{15\left(R_{17}+R_{18}\right)}{R_{16}+R_{17}+R_{18}}\right)-\frac{15 R_{18}}{R_{18}+R_{19}} .
\end{aligned}
$$


According to the piecewise linear function (11) and equation (14), we can choose $R_{16}=12 \mathrm{k} \Omega, R_{17}=2 \mathrm{k} \Omega$, $R_{18}=1 \mathrm{k} \Omega$, and $R_{19}=14 \mathrm{k} \Omega$.

According to Figures 3 and 4, the gird multi-wing butterfly chaotic attractors are obtained via circuit simulation software Multisim 10.0, as shown in Figure 5. When $k_{1}, k_{3}$ are switched on and $k_{2}, k_{4}$, and $k_{5}$ are switched off, the circuit generates $4 \times 2$-wing butterfly chaotic attractors as shown in Figure 5(a). When $k_{1}, k_{3}$, and $k_{4}$ are switched on and $k_{2}$ and $k_{5}$ are switched off, the circuit creates $4 \times 3$-wing butterfly chaotic attractors as shown in Figure 5(b). When $k_{1}, k_{2}, k_{3}$, and $k_{4}$ are switched on and $k_{5}$ is switched off, the circuit generates $6 \times 3$-wing butterfly chaotic attractors as shown in Figure 5(c), and the time series about the state variable $X_{4}$ is shown in Figure 5(e). When $k_{1}, k_{2}, k_{3}, k_{4}$, and $k_{5}$ are switched on, the circuit creates $6 \times 4$-wing butterfly chaotic attractors as shown in Figure 5(d).

From Figures 5 and 1, we can see that the circuit experimental results are in agreement with the results of numerical simulation.

\section{Conclusion}

A new method is presented to construct grid multi-wing butterfly chaotic attractors via nonlinear coupling control in this paper. A five-dimensional grid multi-wing butterfly chaotic system is constructed via this approach. Through adjusting the nonlinear coupling controllers and the piecewise linear functions, the $4 \times 2,4 \times 3,6 \times 3$, and $6 \times 4$ wing butterfly chaotic attractors are obtained. Through the theoretical analysis and numerical simulation, the complex dynamic characteristics of the five-dimensional grid multiwing butterfly chaotic system are shown. Also, the system has been implemented by designing an electronic circuit.

\section{Competing Interests}

The author declares that there is no conflict of interests regarding the publication of this article.

\section{Acknowledgments}

This work is supported by the Technology Research Projects of The Chongqing Education Committee (Grant nos. KJ130509, KJ1400410, and KJ130520).

\section{References}

[1] E. N. Lorenz, "Deterministic nonperiodic flow," Journal of the Atmospheric Sciences, vol. 20, no. 1, pp. 130-141, 1963.

[2] L. O. Chua, M. Komuro, and T. Matsumoto, "The double scroll family. I. Rigorous proof of chaos," IEEE Transactions on Circuits and Systems, vol. 33, no. 11, pp. 1072-1097, 1986.

[3] L. J. Ontañón-García, E. Jiménez-López, E. Campos-Cantón, and M. Basin, "A family of hyperchaotic multi-scroll attractors in $\mathbb{R}^{n}$," Applied Mathematics and Computation, vol. 233, pp. 522$533,2014$.
[4] Y. Lin, C. Wang, and H. He, "A simple multi-scroll chaotic oscillator employing CCIIs," Optik, vol. 126 , no. 7-8, pp. 824$827,2015$.

[5] F. Yu, C. Wang, and H. He, "Grid multiscroll hyperchaotic attractors based on colpitts oscillator mode with controllable grid gradient and scroll numbers," Journal of Applied Research and Technology, vol. 11, no. 3, pp. 371-380, 2013.

[6] C. H. Wang, H. Xu, and F. Yu, "A novel approach for constructing high-order Chua's circuit with multi-directional multiscroll chaotic attractors," International Journal of Bifurcation and Chaos, vol. 23, no. 2, Article ID 1350022, 2013.

[7] A. S. Elwakil, S. Özoğuz, and M. P. Kennedy, "Creation of a complex butterfly attractor using a novel Lorenz-type system," IEEE Transactions on Circuits and Systems. I. Fundamental Theory and Applications, vol. 49, no. 4, pp. 527-530, 2002.

[8] C. Han, S. Yu, and G. Wang, "A sinusoidally driven Lorenz system and circuit implementation," Mathematical Problems in Engineering, vol. 2015, Article ID 706902, 11 pages, 2015.

[9] J. Lü and G. Chen, "A new chaotic attracor coined," International Journal of Bifurcation and Chaos, vol. 12, no. 3, pp. 659-662, 2002.

[10] Q. Han, C.-X. Liu, L. Sun, and D.-R. Zhu, "A fractional order hyperchaotic system derived from a Liu system and its circuit realization," Chinese Physics B, vol. 22, no. 2, Article ID 020502, 2013.

[11] R. Wang, H. Sun, J.-Z. Wang, L. Wang, and Y.-C. Wang, "Applications of modularized circuit designs in a new hyperchaotic system circuit implementation," Chinese Physics B, vol. 24, no. 2, Article ID 020501, 2015.

[12] S. Dadras and H. R. Momeni, "A novel three-dimensional autonomous chaotic system generating two, three and fourscroll attractors," Physics Letters. A, vol. 373, no. 40, pp. 36373642, 2009.

[13] S. Dadras, H. R. Momeni, and G. Qi, "Analysis of a new 3D smooth autonomous system with different wing chaotic attractors and transient chaos," Nonlinear Dynamics, vol. 62, no. 1-2, pp. 391-405, 2010.

[14] J. Liu, "A four-wing and double-wing 3D chaotic system based on sign function," Optik, vol. 125, no. 23, pp. 7089-7095, 2014.

[15] J. Ma, Z. Chen, Z. Wang, and Q. Zhang, "A four-wing hyperchaotic attractor generated from a $4 \mathrm{D}$ memristive system with a line equilibrium," Nonlinear Dynamics, vol. 81, no. 3, pp. 12751288, 2015.

[16] A. Zarei, "Complex dynamics in a 5-D hyper-chaotic attractor with four-wing, one equilibrium and multiple chaotic attractors," Nonlinear Dynamics, vol. 81, no. 1-2, pp. 585-605, 2015.

[17] B. Yu and G. Hu, "Constructing multiwing hyperchaotic attractors," International Journal of Bifurcation and Chaos, vol. 20, no. 3, pp. 727-734, 2010.

[18] S. Yu, J. Lü, X. Yu, and G. Chen, "Design and implementation of grid multiwing hyperchaotic Lorenz system family via switching control and constructing super-heteroclinic loops," IEEE Transactions on Circuits and Systems I: Regular Papers, vol. 59, no. 5, pp. 1015-1028, 2012.

[19] S. Yu, W. K. S. Tang, J. Lü, and G. Chen, "Design and implementation of multi-wing butterfly chaotic attractors via lorenz-type systems," International Journal of Bifurcation and Chaos, vol. 20, no. 1, pp. 29-41, 2010.

[20] M.-W. Luo, X.-H. Luo, and H.-Q. Li, "A family of fourdimensional multi-wing chaotic system and its circuit implementation," Acta Physica Sinica, vol. 62, no. 2, Article ID 020512, 2013. 
[21] S. Yu, J. Lü, G. Chen, and X. Yu, "Generating grid multiwing chaotic attractors by constructing heteroclinic loops into switching systems," IEEE Transactions on Circuits and Systems II: Express Briefs, vol. 58, no. 5, pp. 314-318, 2011.

[22] Y. Huang, P. Zhang, and W. Zhao, "Novel grid multiwing butterfly chaotic attractors and their circuit design," IEEE Transactions on Circuits and Systems II: Express Briefs, vol. 62, no. 5, pp. 496500, 2015. 


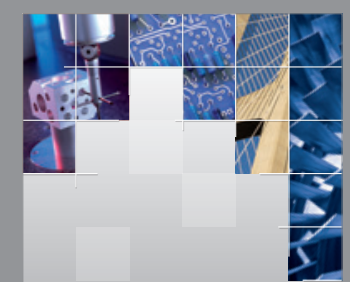

\section{Enfincering}
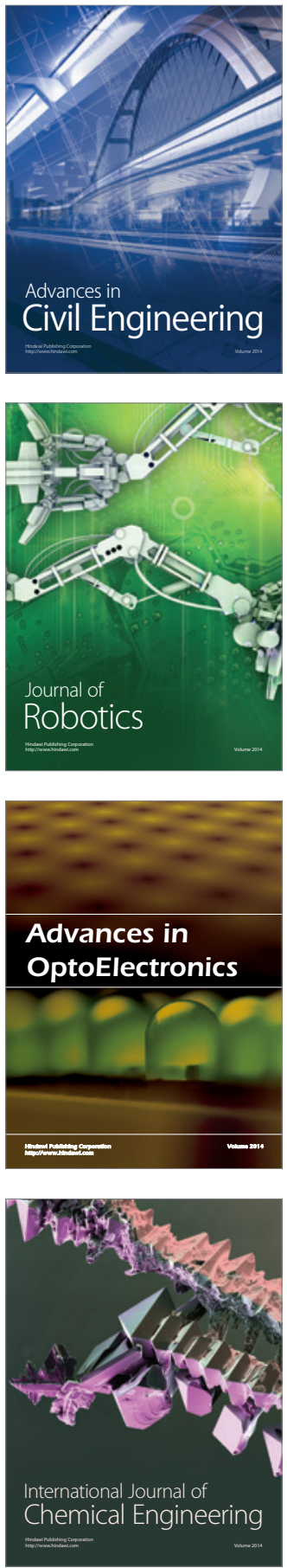

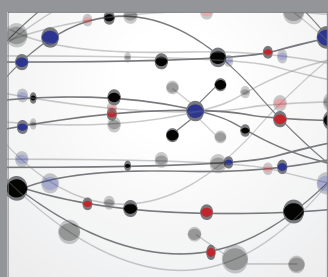

The Scientific World Journal

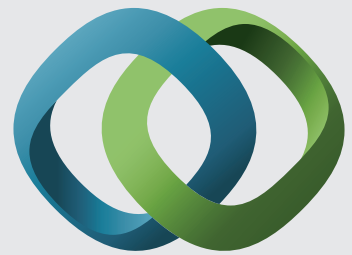

\section{Hindawi}

Submit your manuscripts at

http://www.hindawi.com
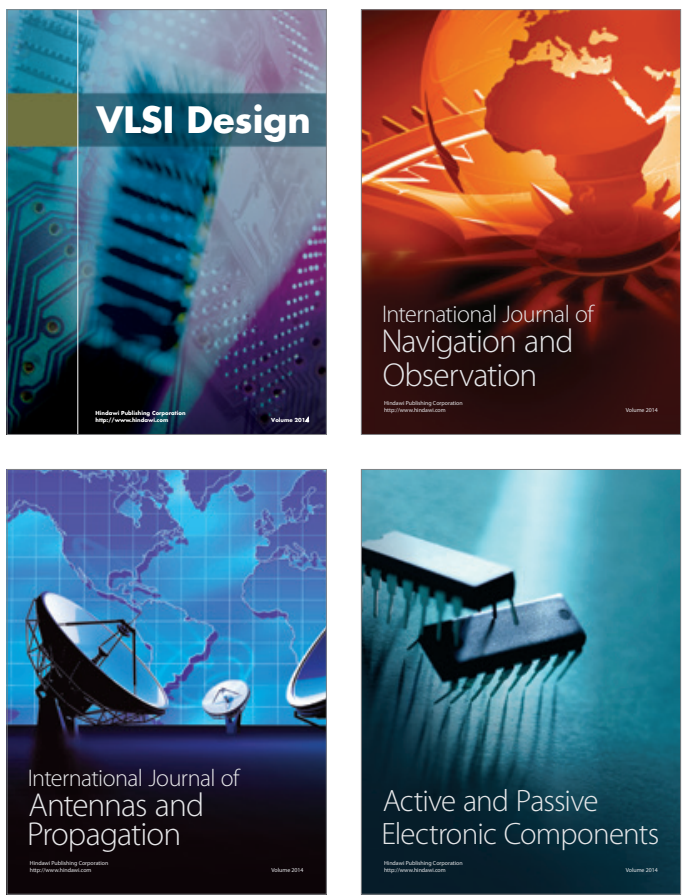
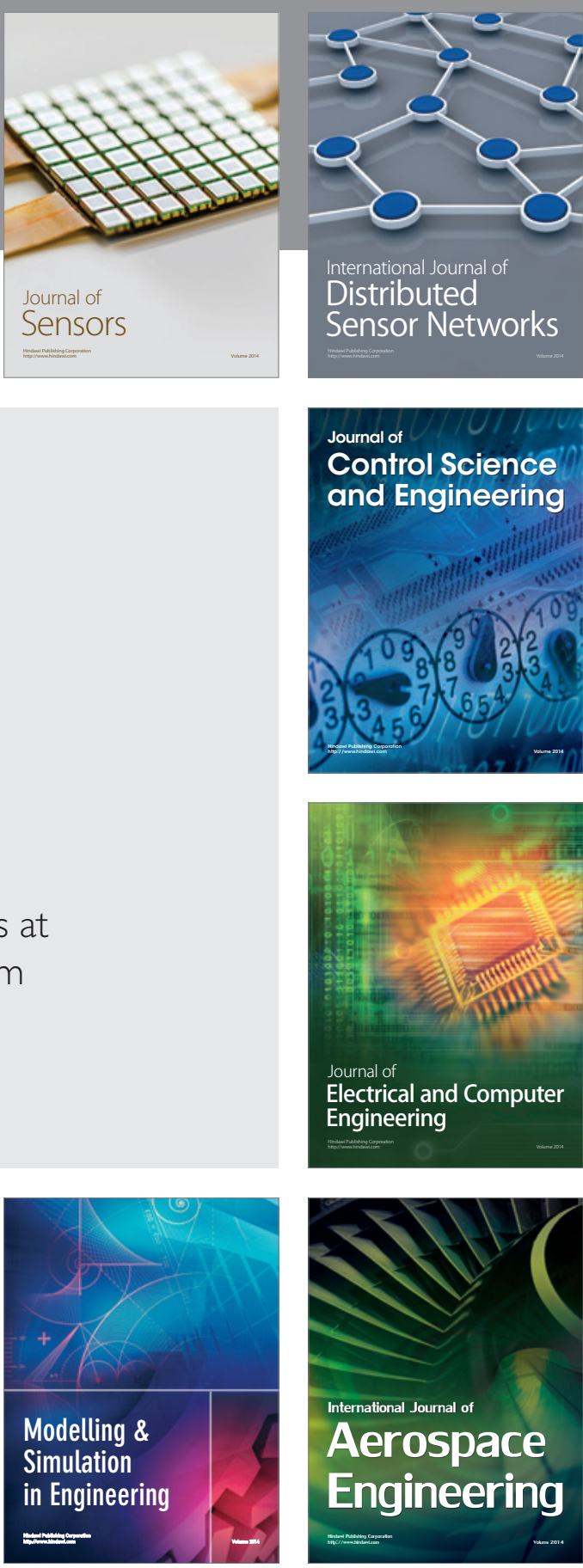

International Journal of

Distributed

Sensor Networks

Journal of

Control Science

and Engineering
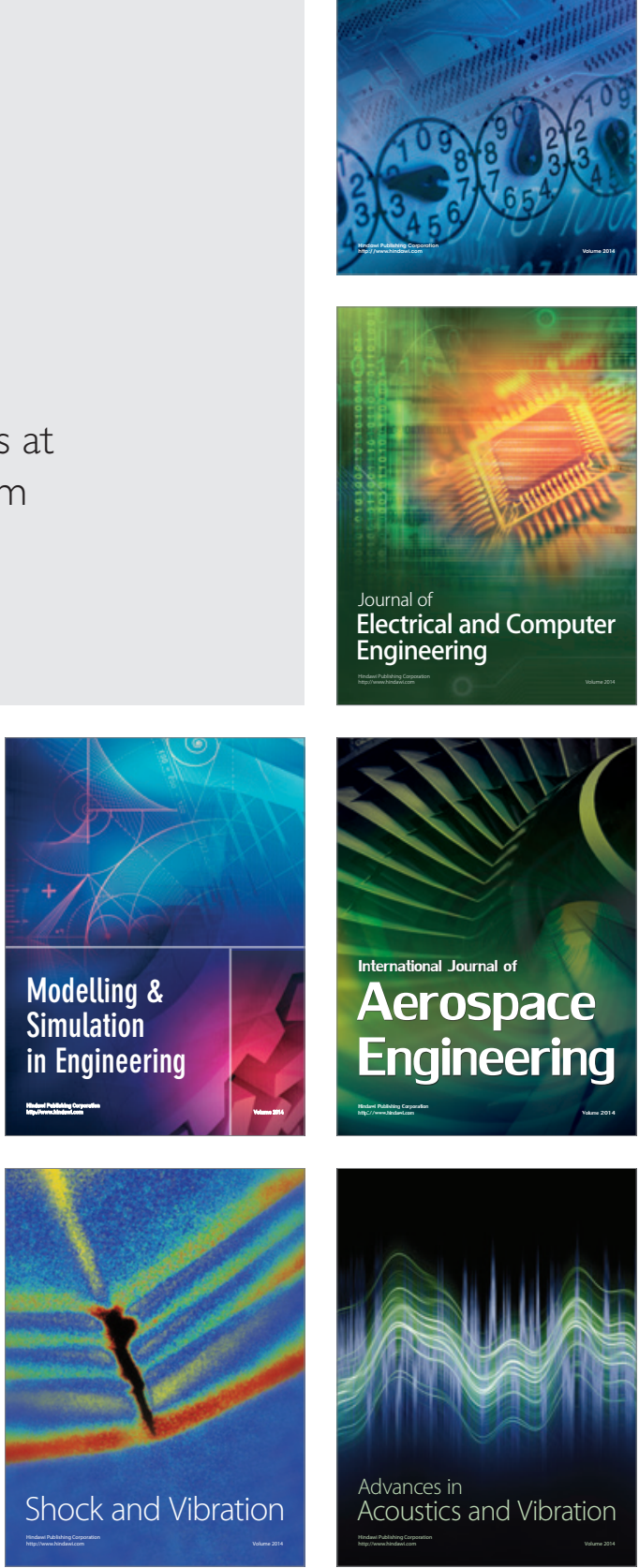